\title{
Responsabilidad, trabajo y condiciones de vida. Problematizaciones sobre los jóvenes en los programas de prevención social del delito en Argentina
}

\author{
Responsibility, work and life conditions. \\ About youths on the social crime \\ prevention's programs in Argentina
}

doi: http://dx.doi.org/10.32870/espiral.v24i68.6332

Emilio Jorge Ayos*

\begin{abstract}
Resumen
El objetivo de este trabajo es analizar bajo qué figuras de la autovalía y la dependencia han sido delimitados los destinatarios de las políticas de prevención social del delito en Argentina durante la primera década del siglo XXI. Se intenta una aproximación a las formas en las que en el presente se reelabora la vieja cuestión de la peligrosidad de los sectores populares y su relación con los modos sociales de problematizar el trabajo $y$ las condiciones de vida, desde un enfoque metodológico cualitativo. Se argumenta que dichas intervenciones tensionan diferentes formas de problematizar las figuras de la autovalía y la dependencia: habilitan un espacio de dependencia legítima ligado a la protección de los derechos de los jóvenes destinatarios $y$, al mismo tiempo, otras que refuerzan los procesos de responsabilización individual en línea con la transformación neoliberal de la política social.
\end{abstract}

Palabras clave: dependencia, jóvenes, trabajo, condiciones de vida, prevención del delito.

\begin{abstract}
The aim of this paper is to analyze how do the recipients of social crime prevention policies are illustrated, particularly under the concepts of self-worth and dependence, in Argentine during the first decade of the new century. We try to know the ways in which the old question of "danger classes" is re-elaborated in our days. Specifically we try to associate such issue whit the ideas of work and life conditions. We use a qualitative methodological approach to analyze the social crime prevention policies in Argentina during the first decade of the new century. We argue that such interventions stressed in different ways the concepts of self-worth and cut dependence: On the one hand enable a rightful self-reliance's space, regarding the protection of the rights of targeted youth; on the other hand other processes enhance self-responsibility, regarding the neoliberal transformation of social policy.
\end{abstract}

Keywords: dependency, youth, work, life conditions, crime prevention.

\footnotetext{
- Profesor-Investigador de la Facultad de Ciencias Sociales de la Universidad de Buenos Aires y del CONICET, Argentina. emilio.ayos@gmail.com

Fecha de recepción: 27 de julio de 2015. Fecha de aceptación: 16 de junio de 2016.
} 


\section{Introducción}

Las políticas de prevención social del delito son aquellas intervenciones que desde el campo del control del delito se enlazan y articulan con el campo de la política social, delimitando un espacio de intersección en el que se encuentran y reorganizan las figuras, los debates y las modalidades de intervención que habitan dichos campos. Y aunque es posible reconocerlos como espacios de intervención diferenciados, sus modos de problematización originarios y sus tensiones constitutivas muestran profundas continuidades: la preocupación por las "clases peligrosas" en los procesos de organización y reorganización del trabajo y las condiciones de vida son comunes tanto a la "cuestión social" como a la "cuestión criminal".

Los programas de prevención social del delito se han caracterizado por hacer del trabajo una de sus preocupaciones centrales. El trabajo, los "problemas sociales" vinculados al trabajo, el desempleo o la no inscripción en las formas "normales" del trabajo asalariado han sido el elemento casi ineludible de las explicaciones sobre las conductas delictivas y de los fundamentos de las políticas preventivas de corte social. En el caso argentino en particular, aunque siguiendo un patrón que puede encontrarse también en el contexto regional, los jóvenes de sectores populares han sido el centro de esta mirada que cruza la cuestión de la "empleabilidad" y las distintas formas de la peligrosidad. En este sentido, los jóvenes de sectores populares son el grueso de las poblaciones objetivo de dichos programas, más allá de que estos sean delimitados formalmente o no como programas hacia "jóvenes".

En este artículo se hace una aproximación a esta cuestión observando las formas en que estos programas de prevención social del delito han reconstruido dos figuras constitutivas del campo de lo social: las de la autovalía y 
la dependencia. Es decir, las construcciones sociales que moldean la tensión entre el mandato de la valía por el propio trabajo individual y las distintas modalidades de dependencia legítima.

En el primer apartado se presenta brevemente el problema que guio la investigación de la que se desprende este artículo, sus lineamientos metodológicos y sus referentes empíricos. En el segundo apartado se analizan las reorientaciones de los campos de la política criminal y la política social en Argentina, de lo cual emergen las orientaciones preventivas. Luego se señala cómo estas diferentes líneas de fuerza moldearon al sujeto-objeto de sus intervenciones en cuanto a la tensión autovalía-dependencia.

\section{Condiciones de vida y delito: problemas, políticas, sujetos}

Tanto la producción social de las condiciones de vida, en la tensión entre trabajo y pobreza que reelabora constantemente la cuestión de la autovalía y la dependencia como forma de dirimir la pertenencia legítima de los sujetos dentro de las sociedades capitalistas, como la producción social del delito, en tanto administración diferencial de los ilegalismos ${ }^{1}$ que constituye a los sujetos de la peligrosidad, tienen un punto álgido de elaboración en las políticas que hacen foco en dichos procesos: las políticas sociales y las políticas criminales, respectivamente.

En este sentido, se entiende a las políticas sociales como la instancia estatal de constitución de la cuestión social en la que se moldean y producen las condiciones de vida de los sujetos, en tanto campo conflictivo en el que se dirime, a su vez, la construcción social de las necesidades, pero también como proceso que tiene como resultado siempre 
inacabado la constitución social del trabajo, de sus sentidos y orientaciones, y de su sujeto, el trabajador, como parte de la producción de formas legítimas de pertenencia social. Esa tensión entre trabajo y pobreza, pensados como conceptos opuestos y excluyentes según la producción social que ha acompañado el desarrollo de sociedades capitalistas, es uno de los puntos centrales sobre el que se elaboran las formas de integración social o la "utilidad en el mundo" de los sujetos, proceso en el que las políticas sociales son protagonistas (Offe, 1990; Castel, 1997, 2004; Grassi, 2003; Danani, 2004, 2010).

La cuestión del delito no ha sido ajena a este proceso: si la pobreza fue progresivamente lo "otro" del trabajo asalariado como forma legítima de satisfacción de necesidades, el delito fue uno de los ejes centrales en su producción social, dado que la pobreza fue construida como signo de peligrosidad, como estigma moral sobre los no "laboriosos".

Ahora bien, el delito no es una entidad transparente, sino que también es una compleja producción social atravesada por procesos históricos de construcción de la desigualdad. Esto se inscribe en una tradición que, aunque diversa, entiende al delito como un constructo social no a partir de dinámicas consensuales, sino como producto de las luchas y los enfrentamientos que estructuran las sociedades. Así, se considera a las políticas criminales o de control del delito como la instancia estatal de este proceso de constitución social del delito, en tanto administración diferencial (Foucault, 1999) del conjunto total de ilegalismos que recorta de ellos una porción producida, así, como "delincuencia", asociada a las prácticas ilegales de los sectores populares, siendo la idea de criminalización central en este proceso de producción de sujetos sociales (Melossi y Pavarini, 1987; Pavarini, 1983; Baratta, 1986; Foucault, 1999). Se dice que estos campos de políticas participan, como instancia estatal, en la producción y moldeamiento de las condiciones 
de vida, para la política social, y del delito, para la política criminal, aunque por supuesto no de manera exclusiva, ya que la producción social de estos ámbitos reconoce formas de problematización que son reconstruidas más allá del espacio estatal.

Las reorientaciones de estos campos de intervención política implican transformaciones en las formas en que son problematizadas las condiciones de vida y el delito, así como sus relaciones recíprocas. Las mutaciones históricas conceptualizadas en torno a la noción de neoliberalismo han afectado fuertemente dichos campos: en relación a las políticas criminales, se ha dado una intensificación punitiva, la emergencia de estrategias "administrativas" y actuariales de control del delito, el descentramiento de la idea de rehabilitación del delincuente y la proliferación de programas inscriptos en el paradigma de la "nueva prevención” del delito (Cohen, 1985; Garland, 1985, 2005; Baratta, 1997; Crawford, 1998, 2009; Sozzo, 2000; Boutellier, 2001; O’Malley, 2004; O’Malley y Hutchinson, 2007; Lea y Young, 2008; Selmini, 2009; Melossi y Selmini, 2009; Ayos, 2010).

Las políticas sociales, por su parte, muestran una tendencia hacia la asistencialización de sus intervenciones, tanto en el sentido de la mayor centralidad de las estrategias asistenciales como de la incorporación de modalidades de gestión y problematizaciones históricamente propias de las estrategias asistenciales en otros sectores de políticas sociales, como la seguridad social o las políticas educativas (Grassi, Hintze, Neufeld, et al., 1994; Cortés y Marshall, 1999; Grassi, 2003; Álvarez Leguizamón, 2006; Soldano y Andrenacci, 2006; Danani y Hintze, 2011; Grondona, 2012).

En conjunto, se pueden conceptualizar estas transformaciones como un proceso de individualización y comunitarización de las formas de intervención social (Danani, 2005a), en tanto estas se atan a las particularidades y los comportamientos subjetivos, o de grupos y territorios 
específicos, problematización en la cual las cuestiones de la inseguridad, la incertidumbre y el riesgo adquieren una preponderancia fundamental (Rose, 1996; Castel, 1997, 2004; Beck, 1998). En Argentina esto se observa con particular intensidad a partir de 1990, mostrando en los últimos años algunas tendencias divergentes, conceptualizadas a partir de la noción de contrarreformas en el campo de la política social (Danani y Grassi, 2008; Lo Vuolo, 2009; Danani y Hintze, 2011; Hintze y Costa, 2011).

En este contexto de individualización y centralización de la cuestión de la inseguridad y el riesgo es donde emerge toda una producción social sobre el retorno de las clases peligrosas, como re-creación de la relación entre condiciones de vida, peligrosidad y delito. Se habla de retorno o de reactualización porque esta producción no es nueva: la idea de las "clases peligrosas" formó parte de la construcción de la "cuestión social" decimonónica, en la que la pobreza (y con ella la cuestión de la asistencia, de la autovalía o la dependencia legítima), como constructo opuesto al trabajo, fue progresivamente asociado a la peligrosidad y al delito (Castel, 1997, 2004; Rose, 1996; Beck, 1998; De Marinis, 1998, 2002; Bauman, 1999; Wacquant, 2000; Pegoraro, 2001), aunque, por supuesto, esta idea sí adquiere novedosas formas actuales de problematización, las cuales son el centro del problema de investigación aquí planteado.

A la vez, en el contexto del neoliberalismo prolifera y cristaliza (aunque sus orígenes son muy anteriores) un espacio específico de elaboración de esta relación entre condiciones de vida y delito, constituyéndose por ello en un lugar privilegiado para el análisis de las nuevas formas de problematización de la cuestión de la peligrosidad de los sectores populares: la corriente de intervenciones que se inscriben en la prevención social del delito.

Este tipo de estrategias se caracterizan por intentar influir en las supuestas causas sociales de los comporta- 
mientos delictivos, es decir, se orienta fundamentalmente hacia los potenciales ofensores y sus condiciones de vida (Baratta, 1997; Sozzo, 2000; Selmini, 2009; Pitch, 2009). Aquí se propone entenderlas como espacios de intersección de la política criminal y la política social, dada la relación histórica que estas políticas preventivas establecieron con las intervenciones enfocadas en las condiciones de vida de los sujetos y los grupos. Esta mirada nos permite interrogarnos acerca de las mutaciones históricas que se observan en los campos de la política social y la política criminal como un eje analítico central para aprehender los procesos en los cuales participan las estrategias de prevención social.

Por último, las políticas de prevención social del delito y las formas particulares en que reconstruyen la relación entre delito y condiciones de vida emergen en el campo de contradicciones y debates políticos que en Argentina, a partir de los años noventa, se estructuraron en torno a la noción de (in)seguridad, siendo parte de las orientaciones (heterogéneas, no cristalizadas en un sentido monolítico) que adoptó la política criminal en el periodo bajo estudio, y por ello la reconstrucción histórica de dicho campo se presenta como marco interpretativo ineludible para analizar las políticas preventivas en sus particularidades históricopolíticas (Font, 1999; Sozzo, 1999; Pegoraro, 2001; Kessler, 2002, 2006, 2008; Saín, 2002, 2008; Guemureman, 2002; De Marinis, 2002; Daroqui, 2003, 2009; Daroqui y Guemureman, 2004; Kaminsky, 2005; Ciafardini, 2006; Isla, 2007; Rangugni, 2009; Galvani, et al., 2010).

En este sentido, se entiende que es necesario historizar la emergencia y el desarrollo de estrategias preventivas en relación a las discusiones sobre la (in)seguridad que se dan en la sociedad argentina, en el marco a su vez de las reorientaciones específicas que se producen en la política social y en la política criminal en dicho país. 
En este punto, se entiende que las políticas de prevención social del delito reactualizan la cuestión de la polaridad seguridad-inseguridad en las sociedades tardo-modernas, poniendo en tensión su problematización en términos de seguridad-inseguridad frente al delito, con la problematización que refiere a la inseguridad social, la proliferación del riesgo o la "liquidez" de las relaciones sociales como características constituyentes de la experiencia vital en el último ciclo histórico.

Por todo ello, un eje fundamental del presente análisis fue inscribir el desarrollo de las políticas de prevención social del delito y la forma en que estas elaboran la relación entre condiciones de vida y delito en el marco de las discusiones políticas y sociales sobre la cuestión de la seguridad que se desarrollan en Argentina en las últimas décadas (Castel, 1997, 2004; Beck, 1998; Rose, 1996; Danani y Grassi, 2008; Pitch, 2009).

A su vez, es importante destacar para el presente problema de investigación que la problematización centrada en la idea de inseguridad ha realizado una cesura en cuanto a la producción de sujetos sociales, fijando los sentidos a partir de los cuales se perciben y distinguen socialmente los sujetos amenazantes y amenazados, victimarios y víctimas. Esta producción ha sido identificada por variadas investigaciones, señalando que los jóvenes, varones y de sectores populares son los portadores de toda una construcción social que los performa como los sujetos-objetos de la inseguridad en tanto agentes amenazantes, peligrosos, violentos (Guemureman, 2002, 2011; Daroqui y Guemureman, 2001, 2004; López, et al., 2011; Calzado, 2004; Pegoraro, 2002; Vilker, 2011).

Aunque, como se vio, la cuestión de la delincuencia juvenil no es un elemento nuevo en el campo de la política criminal, y en Argentina la conformación de una "justicia de menores" se observa en las primeras décadas del siglo XX, 
se asiste a un reforzamiento de una división simbólica tajante entre grupos: por un lado están los "ciudadanos" respetables y por otro los sectores "peligrosos" o desviados, reconstruyendo una imagen de orden social en el que existen valores, normas e intereses comunes a la totalidad de los ciudadanos y al Estado, como su expresión y garante, y también existen estos grupos como una amenaza exterior que cuestiona dichos valores comunes, actualizando una noción de defensa social (Baratta, 1986; Daroqui, 2003). Estos sentidos son impugnados por diferentes argumentaciones desde sectores diversos, aunque conservan una intensa capacidad para delimitar los debates sociales.

En este contexto, la cuestión de la juventud ha sido uno de los ejes centrales de la producción de las ciencias sociales sobre la (in)seguridad, reconstruyendo como problema de investigación, bajo las modalidades propias del campo de la investigación científica y académica, esta cuestión central en la agenda pública durante el periodo. Los estudios han identificado las formas en que se imbrican nuevas dinámicas sociales, nuevas prácticas e identidades juveniles con los microdelitos callejeros, es decir, con esa parte predilecta de las agencias penales (Rossini, 2003; Isla, 2002; Míguez, 2002; Kessler, 2002, 2006; Tonkonoff, 2003, 2007a, 2007b).

Se analizan aquí entonces las políticas de prevención social del delito durante el periodo 2000-2010 en su intersección con el campo de las políticas sociales. La periodización encuentra su punto inicial en la creación del Plan Nacional de Prevención del Delito, en el año 2000, en un contexto en el que la cuestión de la "inseguridad" había alcanzado niveles de politización hasta entonces inéditos (Sozzo, 2008). El punto final de la periodización se estableció con la creación del Ministerio de Seguridad en el año 2010, con la que se expresó una reorientación de las líneas de fuerza que organizan el campo de las políticas criminales, como luego se analizará. 
De esta manera, se seleccionaron tres intervenciones que en su desarrollo se vincularon con el Programa de Empleo Comunitario (PEC) (Ministerio de Trabajo, Empleo y Seguridad Social): el Programa "Comunidades Vulnerables" (Dirección Nacional de Política Criminal), los programas municipales "Post-Comunidades Vulnerables" y la "Intervención multiagencial para el abordaje del delito en el ámbito local" (Secretaría de Seguridad Interior/Programa de Naciones Unidas para el Desarrollo).

El PEC fue creado por el Ministerio de Trabajo y Seguridad Social (MTEYSS) en el año 2003, como cristalización de experiencias originadas al interior del "Programa de Emergencia Laboral" (PEL), iniciado en 1999, y en el marco de la aparición del "Programa Jefas y Jefes de Hogar", en el año 2002. En el contexto de la declaración de la emergencia social, económica, administrativa, financiera y cambiaria por parte del Gobierno nacional, el MTEYSS decidió abrir la incorporación al PEL de sectores que quedaban por fuera de la delimitación de la población-objetivo del Programa Jefas y Jefes de Hogar, especialmente los jóvenes. En ese sentido, se dispuso un monto del beneficio similar al programa anterior (ARS 150), y, como contraprestación, la realización de actividades "comunitarias" de carácter mensual, esto para 300000 beneficiarios. A partir de esta experiencia anterior dentro del PEL, nació en enero del 2003 el PEC, con el objetivo de "promover la participación de trabajadores desocupados en situación de vulnerabilidad social en proyectos que mejoren su empleabilidad y faciliten su inserción laboral" (MTEYSS, s/p).

En segundo lugar, este trabajo se aboca al caso del programa "Comunidades Vulnerables" (PCV), por ser la experiencia más importante en cuanto a tácticas de prevención social del delito dentro del ámbito nacional argentino, esto hasta su finalización, en el año 2008. Este programa surgió en el año 2001 como parte del naciente Plan Nacional de Prevención del Delito (PNPD), recuperando experiencias 
anteriores -ligadas al "Plan Alerta", en la ciudad de Buenos Aires-. Entre esos años, se aplicó en diferentes puntos de la ciudad de Buenos Aires y el Gran Buenos Aires (como Morón, Avellaneda, Tres de Febrero, San Isidro, entre otros), además de hacerlo en otras provincias: Río Negro, Neuquén, Chubut, Mendoza y Santa Fe. Su implementación se presentó como focalizada en barrios de alta vulnerabilidad social, abocada a dos ejes de acción interrelacionados: 1) la prevención de la violencia social; y 2) la prevención del delito.

El hecho fundamental que sustenta la selección del PCV en el presente trabajo se relaciona con el particular vínculo que el mismo estableció con el campo de las políticas sociales: la vinculación histórica del PCV con los planes asistenciales administrados por el Ministerio de Trabajo, Empleo y Seguridad Social, y, específicamente desde el año 2003, con el Programa de Empleo Comunitario (PEC). El otorgamiento de estos planes fue un factor fundamental de las estrategias de prevención social del delito implementadas por el PCV, puesto que el beneficio correspondiente al PEC se otorgaba a su "población-objetivo", con la condición de participar en sus dispositivos de intervención. En este sentido, los programas de prevención social del delito se constituían en la contraprestación requerida para percibir el beneficio otorgado por el PEC, como luego se analizará.

En tercer lugar, se seleccionaron las intervenciones municipales que, al finalizar el PCV, en el año 2008, continuaron implementando localmente las estrategias de prevención "social” del delito que articulaban con el PEC. Se hace referencia a los casos en los que las intervenciones locales que funcionaban en el marco del PCV prosiguieron sus prácticas de gestión sobre las poblaciones-objetivo seleccionadas, aunque la continuidad o la ruptura de las lógicas de intervención es un interrogante que se aborda en esta investigación. 
Por último, se analiza el programa de "Intervención multiagencial para el abordaje del delito en el ámbito local" (PIM) (Secretaría de Seguridad Interior/PNUD), durante los años 2008-2010. El mismo tiene como objetivo "promover la implementación de políticas integrales de seguridad con énfasis en la prevención que, sin desvalorizar los aportes que pueden realizar las instituciones policiales, incluyan y comprometan a otras agencias gubernamentales y actores no gubernamentales en el abordaje y resolución del problema" (PIM, 2008, s/p).

En principio, el PIM se enfocó sobre tres ciudades medianas, aunque sólo pudo instrumentar intervenciones de prevención social territoriales en una ciudad de la provincia de Santa Fe. Se estructuró en dos etapas: una primera, abocada al diagnóstico de la situación a partir del análisis documental de los programas estatales sobre la materia y de entrevistas en profundidad con actores claves (tanto estatales como de la sociedad civil), y una segunda etapa en que se tomó dicho diagnóstico y se elaboró una estrategia multiagencial de abordaje de problemas prioritarios de seguridad para dichas ciudades. La inclusión de esta intervención en el plan de trabajo de la presente investigación se debe a que es la única política a nivel nacional que desde el año 2008 integra estrategias de prevención social, y en este sentido no es casual que ubique explícitamente como su principal antecedente al PNPD, al cual pertenecía, como se ha dicho, el PCV.

La presente investigación adoptó un enfoque metodológico cualitativo. Dados los diferentes ejes que recortan el problema de investigación, se han diferenciado tres planos de indagación empírica y reconstrucción analítica: el de las condiciones sociopolíticas e institucionales generales; el que refiere a la cuestión específica de la relación entre condiciones de vida y delito, en términos de sus modos de problematización; y, por último, la dimensión de las formas 
de problematización que circulan en el desarrollo de políticas específicas de prevención social del delito en Argentina durante la primera década del siglo XXI. Sobre la selección de los desarrollos de estos programas en diferentes unidades político-administrativas y de gestión, la investigación combinó el uso de fuentes primarias (entrevistas en profundidad a funcionarios y operadores de los programas analizados) y fuentes secundarias de diversos tipos, acordes a los diferentes planos de indagación empírica que se han señalado.

Para la construcción de la muestra se utilizó la estrategia del muestreo teórico (Glaser y Strauss, 1967). Se adoptó con este fin el criterio de saturación teórica, es decir, se incluyeron distintas unidades de relevamiento hasta el momento en que las construcciones de sentido en torno a la problematización de la relación entre condiciones de vida y delito que surgieron de ellos no aportaron particularidades nuevas (Glaser y Strauss, 1967; Soneira, 2004). Se consideraron unidades de relevamiento a los funcionarios e integrantes de los equipos profesionales de los programas en cuestión (a partir de la técnica de entrevistas abiertas), y los documentos que conformaron el corpus (utilizando técnicas de investigación documental).

En cuanto a las primeras, se realizó la selección distinguiendo tres grupos de individuos: en primer lugar, las "autoridades", es decir, las personas que comandaban las políticas en el nivel nacional de gestión. En segundo lugar, las "autoridades locales", quienes fueron los funcionarios locales inmediatamente a cargo de la gestión local de las intervenciones. Y por último, los "operadores", los cuales se diferenciaban por ser los agentes que llevaban a cabo las tareas previstas en las políticas y que tenían un contacto directo con los territorios de intervención y la "población objetivo" que definía el programa.

$\mathrm{Al}$ caracterizar a los individuos entrevistados, uno de los elementos que sobresalió fue el alto nivel de instrucción 
en cuanto a la educación formal: todos los entrevistados son profesionales universitarios. Pertenecían a tres áreas profesionales: las ciencias sociales (trabajadores sociales, sociólogos o politólogos), el derecho (abogados) y la psicología. En el caso de los operadores, eran más numerosos los trabajadores sociales y los psicólogos. Este grupo presentó otra característica fundamental, ligada al género: la gran mayoría de los operadores fueron mujeres. Por último, en cuanto a la pertenencia territorial, si bien la mayoría de los operadores vivía en el partido o municipio donde se instrumentaban los programas, y esto les otorgaba cierto conocimiento acerca de las zonas específicas de intervención, ellos no vivían en las mismas, es decir, no eran operadores que habitaban en el "barrio" donde trabajaban.

Se realizaron un total de treinta y nueve entrevistas a profundidad. El trabajo de campo se llevó a cabo desde mediados del año 2006 hasta finales del año 2009, y luego desde finales del año 2010 hasta finales del año 2011. La distribución de las entrevistas fue la siguiente: nueve del total fueron realizadas a autoridades de los planes y programas analizados, cinco a autoridades locales en la gestión de los mismos, $\mathrm{y}$ veinticinco a operadores territoriales. Se ha mantenido la proporción entre las experiencias de los dos municipios seleccionados en las entrevistas a autoridades locales y operadores y en las intervenciones del PCV y los programas post PCV. El caso del PIM es diferente, dado que un solo agente se configuró como el único "operador" de la acotada intervención territorial que pudo desplegar este programa en la ciudad seleccionada. El mismo fue entrevistado.

\section{I. Políticas sociales y prevención del delito: responsabilidad, autovalía, dependencia}

Las diferentes modalidades que han dibujado históricamente las intervenciones estatales de política social, desde la tradición asistencial hasta las lógicas de la seguridad 
social, marcan en términos generales divergencias en cuanto a sus formas de problematizar los sujetos de la intervención. Uno de los elementos centrales que se pone en juego en estos procesos es la cuestión acerca de la responsabilidad de los sujetos respecto a sus condiciones de vida y su relación con el trabajo. Es decir, tradiciones como la asistencia o la seguridad social proponen diferentes formas de entender las condiciones de vida de los sujetos y las modalidades e instituciones que regulan la responsabilidad sobre dichas condiciones de vida, dejando de lado por un momento las múltiples orientaciones históricas que las mismas han adquirido en diversas latitudes y contextos nacionales.

En estas diferentes formas en que expresan los arreglos institucionales sobre la responsabilidad de los sujetos por sus condiciones de vida se producen las condiciones y los límites en que las sociedades capitalistas definen el imperativo de la autovalía, vinculado a organizar las condiciones de vida alrededor del propio trabajo y, como contracara, las formas de la dependencia y sus patrones de legitimidad. Estas modalidades de la dependencia se delimitan no sólo por los límites de la distinción trabajo/no trabajo (como podría interpretarse, por ejemplo, el tipo de dependencia legítima que adquieren los niños en edad escolar, aunque no sin complejidades), sino también por las dimensiones de las condiciones de vida de los trabajadores y sus familias que son socializadas en diversas formas y grados por las intervenciones estatales de política social y, lo que es central, sobre los distintos contenidos político-culturales que reconstruyen.

Para decirlo más claramente: es evidente que la sustracción de los niños en relación a la obligación del trabajo es una dependencia "legítima" sustentada en la apelación a sus derechos inalienables. Sin embargo, es posible analizar las relaciones entre autovalía y dependencia en procesos no tan tajantes: en el límite, la cobertura de salud de la familia de 
un trabajador bajo la lógica de la seguridad social no proviene directamente del intercambio de los frutos del trabajo en el mercado, sino que supone un andamiaje institucional que convoca la participación de otros "interdependientes", y aquí también los patrones de legitimidad que se construyen para sostener esta "dependencia" pueden invocar desde los contenidos político-culturales ligados al "derecho de ciudadanía" hasta la pura y dura "contribución" individual, y su asimilación con los seguros privados.

Si las políticas sociales se insertan en estos procesos de producción de las condiciones de vida y también de producción de sujetos sociales, en cuanto a sus responsabilidades sobre las mismas en tanto sujetos "autoválidos" e investidos de diversas "dependencias", los programas de prevención social del delito (en tanto espacio de intersección entre los campos de la política criminal y la política social) participan de estos procesos de producción de lo "social" de un modo particular. En ellos se adiciona la participación en la producción de ese constructo social que conocemos como delito y de los sujetos asociados al mismo, bajo la figura histórica del delincuente. En las políticas de prevención social del delito es posible observar una producción acerca de cómo se entiende el delito, atravesada a su vez por cómo lo social es problematizado; una relación entre el delito y lo social que no es por supuesto unívoca, y que ha sido tratada de maneras diversas tanto en las diferentes producciones de la teoría criminológica como en las diferentes experiencias históricas de política criminal.

De esta manera, las políticas de prevención social del delito participan no sólo en los procesos de definición de las modalidades de responsabilidad de los sujetos por sus condiciones de vida (en tanto articulación con las políticas sociales), sino que también lo hacen en los procesos de definición de las formas de responsabilidad que se asignan a los sujetos sobre las prácticas definidas como delictivas. 
Y no lo hacen desvinculadamente: lo social aparece en el centro de su problematización sobre la asignación de la responsabilidad de los sujetos sobre las prácticas delictivas.

Condensando lo dicho hasta aquí: las políticas de prevención del delito reactualizan de maneras variadas la vieja cuestión de las "causas sociales" de los comportamientos criminales; ellas intentan intervenir sobre esa dimensión social que observan como origen último del delito. Sin embargo, como vimos, lo social no es un constructo homogéneo ni constante: las "dependencias" que producen varían en sus formas y en los contenidos político-culturales que las sustentan. Estas diferentes formas de problematización moldean a su vez los modos en que las intervenciones preventivas construyen a sus sujetos. En este sentido, las orientaciones que atraviesan el campo de la política social con las que las políticas preventivas se "encuentran" son constitutivas de sus propias intervenciones.

2. Reorientaciones y entrecruzamientos de los campos de la política social y la política criminal

En este apartado, se presentan sucintamente las dinámicas históricas y relaciones que trazaron los campos de la política social y la política criminal en Argentina. Estas marcaron las orientaciones de los programas de prevención social del delito y las formas en que produjeron una particular manera de construir a su población objetivo. Para analizar las dinámicas que estructuraron históricamente en campo de la política social en Argentina, se propuso como clave interpretativa general la tendencia hacia la centralización y descentralización de la seguridad social y la asistencia, entendidas estas no sólo como "sectores" de políticas diferentes, sino como grillas de inteligibilidad que reconocen vertientes diversas. 
Aunque se propone entender lo "social" como un constructo siempre compuesto en su interior, se sostiene que, en términos globales, su construcción a lo largo del siglo $\mathrm{XX}$, sobre todo desde la década del cuarenta, tuvo como una de sus tendencias organizadoras la centralización de la seguridad social y de sus modalidades de intervención asociadas. A contrapelo de este proceso, la reorientación que comenzó desde mediados de los años setenta, y que se profundizó drásticamente durante la década de los noventa, puede globalmente interpretarse como un movimiento de descentramiento de aquella matriz y de la centralidad de la asistencia, que permeó el campo de la política social más allá del sector de las políticas asistenciales propiamente dicho.

En esta reconfiguración del campo de la política social en Argentina se ha inscripto la articulación con el campo de la política criminal que conforman las estrategias de prevención social del delito. Se interpreta que las nuevas modalidades y orientaciones que presenta la política social abrieron el espacio de confluencia con la política criminal (al mismo tiempo que esta, por supuesto, también se reorganizaba). La creciente centralidad de intervenciones inscriptas en el ámbito de la asistencia, en las diversas formas en que este espacio se estructuró sobre la dimensión subjetiva individual de los destinatarios (en sus comportamientos, sus (in) capacidades, sus stocks de capitales, su (in)empleabilidad, su racionalidad para prever el futuro y encarar proyectos de vida), se articuló estratégicamente con la preocupación de las estrategias preventivas por los "grupos en riesgo" y los comportamientos delictivos.

Ahora bien, esta interpretación global se asienta en la identificación de ciertas líneas de fuerza específicas que atravesaron dicha mutación en el campo de la política social. En el ámbito de las políticas asistenciales, se ha aislado la vertiente del workfare (en la cual se inscribió el PEC) y su preocupación por las capacidades subjetivas que 
constituían a la (in)empleabilidad de los sujetos y grupos específicos, y que además presentaba como forma de condicionalidad particular la contraprestación laboral o de capacitación laboral, que fue el punto de encuentro concreto con las políticas preventivas: ellas se construyeron como la contraprestación que debían cumplir los destinatarios para acceder al beneficio que otorgaba el PEC.

Asimismo, también se identificó otro eje (entramado con los desarrollos del workfare) sobre el cual la prevención social del delito hizo pie: la reactualización del desarrollo comunitario orientada hacia la activación de las energías comunitarias en tanto responsabilización de sus sujetos e instituciones sobre sus condiciones de vida, y que también apela a las capacidades, a las motivaciones y a los patrones culturales que "recortan" estos territorios. La misma presenta continuidades con las propuestas de desarrollo comunitario que proliferaron en el contexto del desarrollismo a partir de finales de la década de 1950, como la cuestión de las “zonas" específicas o la problematización de la "marginalidad" en un registro cultural, pero también significativas diferencias: ese enfoque "estructural", la relevancia de la "nación" y el "pleno empleo", la dualidad y la heterogeneidad como problema a superar (Grassi, Hintze, Neufeld, et al., 1994; Grondona, 2012). Al mismo tiempo, esta articulación con las estrategias preventivas se sustentó en la relevancia que la tradición del desarrollo comunitario tuvo en la propia "nueva prevención" del delito y en algunos de sus antecedentes (Ayos, 2014).

El último movimiento que se identificó en el campo de la política social fue el que mostró ciertas tendencias hacia una recentralización de la matriz de la seguridad social desde mediados de la última década. En Argentina, esta tendencia tuvo uno de sus puntos más intensos en la creación de la Asignación Universal por Hijo para la Protección Social (AUHPS), en el año 2009, que además reconfiguró la relación 
entre seguridad social y asistencia, central para el presente problema de investigación. La misma implicó la creación de un nuevo componente no contributivo al sistema tradicional de la seguridad social, bajo la mecánica de la extensión de las asignaciones familiares (hasta ese momento exclusivas para los trabajadores formales) hacia los trabajadores informales y los desocupados, reconfigurando el sujeto de la seguridad social clásica en Argentina (Hintze y Costa, 2011 y 2014).

Se ha ubicado el surgimiento de la AUHPS y el desdibujamiento que ella produce en las modalidades tipo workfare (incluido el PEC, alejándose de la contraprestación en tanto modo de condicionalidad) como unas de las condiciones de posibilidad que abre el proceso de desacoplamiento entre los campos de la política social y la política criminal en las formas en que este se había configurado en las estrategias preventivas durante la última década. La entrada del PEC en su etapa de finalización luego de la creación de la AUHPS, lo que implicó la clausura de nuevas "altas" en los programas preventivos, significó uno de los elementos que generaron un progresivo debilitamiento de estas intervenciones que imposibilitaron continuar con las modalidades que los estructuraron durante el periodo estudiado.

De esta manera, si la descentralización de la seguridad social y la preponderancia de la asistencia (en las formas particulares del workfare y el desarrollo comunitario) abrieron la superficie de contacto en el campo de la política social para la articulación estratégica con el campo de la política criminal en las estrategias de prevención social, la tendencia a la recentralización de la seguridad social y la reconfiguración de su relación con la asistencia marcan la obturación de ese espacio y la promoción de un desacople de dicha articulación. Por supuesto, esto en el plano de las políticas estatales, dado que las transformaciones en el mercado de trabajo también son un factor fundamental 
para entender la declinación de los candidatos al workfare, aunque esta cuestión escape a este trabajo.

Por supuesto, estos movimientos no implican sólo a las modalidades que adquiere la política social: ellos se inscriben a su vez en los procesos históricos que organizaron el campo de la política criminal en Argentina. La introducción de la tensión seguridad-inseguridad sobre la cuestión del delito y su administración durante la década de 1990 fue expresión de una verdadera reorganización de la política criminal, produciendo nuevos sujetos y objetos, aunque con líneas de fuerza diversas y discursos polémicos, es decir, constituyéndose como un campo de enfrentamientos.

Estas tendencias muestran la centralización de una sensibilidad que intensifica los contenidos punitivos, que es crítica de la justicia penal por su permisividad y por "atarles" las manos a las fuerzas de seguridad, y que desde un posicionamiento emotivo y moral no duda en fijar como sujetos productores de esta inseguridad a los jóvenes, varones, pobres, habitantes de villas o asentamientos, aunque, como dijimos, en un campo que es múltiple y conflictivo.

La orientación preventiva en la Argentina nació en el espacio conflictivo que se estructuró en torno a la (in)seguridad, específicamente en el contexto en el que su politización se intensificaba a niveles inéditos en el pasado. Se hace referencia a las discusiones que se entramaron a partir de las campañas electorales del año 1999, en las que la cuestión del delito tuvo una inusitada centralidad y en las cuales surgió fuertemente el discurso de la "mano dura" que hiciera suyo el candidato y luego gobernador por la provincia de Buenos Aires por el Partido Justicialista, Carlos Ruckauf. No obstante, la virulencia del posicionamiento de Ruckauf no debe ocultar que los rasgos punitivos se extendieron más allá de su espacio político, provocando un proceso de reforma legislativa de endurecimiento penal. 
En este contexto, se interpreta el lanzamiento del PNPD como un posicionamiento que intenta explícitamente polemizar con esta mirada de la "mano dura" desde la propuesta de una orientación preventiva en oposición a las soluciones "represivas". La estructura argumentativa que organiza este posicionamiento presenta las siguientes marcas: en primer lugar, una construcción en un registro científicotécnico que entendía a la prevención como la intervención que podía atacar las verdaderas "causas" del delito; en segundo lugar, una dimensión ético-política, que como parte de un posicionamiento progresista, veía a la orientación preventiva como parte de un proyecto que pugnaba por una política democrática de seguridad, que mediante un "giro a lo social" y un fomento de la participación ciudadana, consideraba la seguridad en el marco de los valores e instituciones democráticas, a diferencia de la respuesta policial y represiva.

La orientación inicial del Gobierno de Néstor Kirchner puede interpretarse en este contexto de posicionamientos que resisten o se oponen a los contenidos más punitivos que atraviesa la problematización sobre la (in)seguridad, aunque esta orientación se desdibujara en el marco de los procesos políticos que desencadenó el "caso Blumberg" en el año 2004. Este fue el segundo momento de más alta politización de la (in)seguridad, y disparador de otra "oleada" de

2. En torno al secuestro y asesinato de Axel Blumberg en el año 2004 se produjo una intensificación en cuanto a la politización de la "inseguridad" con claros contenidos punitivistas que abría un desarrollo y hasta una masificación de los procesos de movilización y reclamo social sobre la "inseguridad" realmente inéditos en Argentina, instalando el miedo al delito en el contexto urbano como la preocupación pública central (Calzado y Van Den Dooren, 2009). Con la consigna "Cruzada Axel, por la vida de nuestros hijos", el 0I de abril del 2004 se realizó la primera de una serie de marchas y movilizaciones públicas que se distinguieron por su masividad (los medios de comunicación estimaron de 100000 a 150000 personas en la primera de ellas), que expresaron una sensibilidad social que cristalizaba en el liderazgo de Juan Carlos Blumberg en tanto padre-víctima (Murillo, 2008; Calzado y Van Den Dooren, 2009). 
reformas legislativas de endurecimiento penal. Este amague progresista (Saín, 2010) significó también la finalización de cierto apoyo político a las intervenciones preventivas que realizaban el PNPD y el PCV, y más en general, a la Dirección Nacional de Política Criminal, lo que se reflejó en las dificultades iniciadas con la producción y difusión de las estadísticas que esta agencia realizaba.

La creación del Ministerio de Seguridad en el año 2010 significó un quiebre de esta tendencia que se iniciara a mediados del año 2004, y encontró uno de sus antecedentes inmediatos en la conformación de cierto entramado crítico frente a las orientaciones de la política criminal del periodo, entramado que fue plasmado en el "Acuerdo por una seguridad democrática", que volvía sobre esa dimensión éticopolítica centrada en la idea de una seguridad democrática que había enmarcado el lanzamiento del PNPD. El contexto inmediato de creación del Ministerio de Seguridad también recuperó estos sentidos, anudando la idea de seguridad a la de derechos humanos en tanto posicionamiento democrático, en el marco del asesinato de Mariano Ferreyra ${ }^{3}$ y los sucesos del Parque Indoamericano. ${ }^{4}$

Ahora bien, este posicionamiento por una política democrática de seguridad hizo pie en dos ejes: el control político de la seguridad, particularmente de las fuerzas policiales, y la promoción de la participación comunitaria, interpelando a sujetos colectivos, políticos y sociales. La recuperación de la prevención del delito (incluida la prevención social) no

3. Joven militante del Partido Obrero asesinado en octubre de 2010 durante una manifestación en contra de la precarización laboral de los trabajadores ferroviarios, crimen por el cual fueron condenados el líder de la Unión Ferroviaria y otros integrantes del sindicato, además de seis oficiales de la policía federal por "liberar" la zona y no intervenir.

4. Represión policial suscitada ante un conflicto social en torno a la cuestión de la vivienda por toma de tierras en el Parque Indoamericano de la Ciudad Autónoma de Buenos Aires, en diciembre del año 20 I0, en la que participó la policía federal $y$ en la que fueron asesinadas tres personas. 
fue ajena a las formulaciones y documentos programáticos del nuevo ministerio; sin embargo, se observó una decisión de no avanzar en esa línea de intervención, descentrando a la prevención social del delito de su desarrollo. Este descentramiento de la idea de prevención social del delito se correspondió con el debilitamiento y la casi absoluta desaparición de las estrategias de prevención social del delito que en Argentina intentaron tener cierta envergadura o alcance nacional: en el año 2010, junto con la emergencia del Ministerio de Seguridad, se desdibujaron las intervenciones post PCV y el PIM.

De esta manera, se observa que en el mismo momento, en el mismo proceso sociopolítico en el que se desdibujaron los lineamientos del campo de la política social que habían abierto una superficie de articulación estratégica con las orientaciones preventivas, en el campo de la política criminal surgió un nuevo proyecto de una "seguridad democrática”, y se observa también que, aunque retomaba en sus programaciones la cuestión de atacar las "causas sociales" con estrategias preventivas, no estableció su desarrollo. Así, se observa que la primera década del nuevo siglo se abrió y se cerró con dos proyectos por una política democrática de seguridad: el primero de ellos centrado en el desarrollo de estrategias preventivas que contuvieran, como elemento interno, un hincapié en los procesos de participación ciudadana; el segundo ubicando como ejes prioritarios el control político de las fuerzas policiales y la promoción de la participación, interpelando a organizaciones políticas y sociales.

\section{Los jóvenes y las formas de la dependencia}

En este apartado se señalan algunos de los ejes a partir de los cuales se puede reconstruir la forma en que los programas de prevención social en Argentina concibieron a los jóvenes en conflicto con la ley penal en cuanto a la tensión

\section{2}


entre autovalía y dependencia, y, como era de esperarse, no por casualidad, sino por la naturaleza misma de las intervenciones estatales, esta construcción es compleja y contiene producciones político-culturales en tensión.

En primer lugar, se pueden observar un conjunto de orientaciones promovidas por estas políticas que construyen un espacio de "dependencia" fuertemente legitimado en términos de la protección de los derechos de estos sujetos y en reparación de las situaciones de vulnerabilidad a las que están expuestos los mismos, tanto en términos de vulnerabilidad social como en relación a su vulnerabilidad penal (y cierta ambigüedad entre ambas -Ayos, 2013-).

En los lineamientos que mostraban los documentos de diseño de estas intervenciones, se pudo observar que las mismas recuperaban un conjunto de conceptualizaciones y problemas que se pueden inscribir en términos generales a una perspectiva "crítica" del saber criminológico. De esta manera, se observa cómo estas intervenciones hacían hincapié en tener en cuenta los procesos de criminalización sobre los sectores populares, reconociendo por ejemplo la existencia del "delito de cuello blanco".

A la vez, retomaban modalidades y nociones que desde estas perspectivas "críticas" se habían realizado específicamente en relación a la prevención del delito, como la idea de prevención integrada. Esta estaba ligada a la experiencia italiana, influida por las discusiones de los criminólogos "críticos" italianos con los realistas de izquierda británicos (Selmini, 2009; Ayos, 2014).

Expresando esa orientación "social" en el marco de un posicionamiento "progresista" frente a las políticas de "mano dura" que se repasaron en el apartado anterior, estas políticas manifestaban explícitamente sus objetivos en términos de producir prevención del delito a partir de proteger a los jóvenes destinatarios mejorando sus condiciones de vida y su situación de vulnerabilidad, entendida como verdadera 
"causa social" del delito. Es decir, la intervención intentaba constituir un espacio de "dependencia", dada la asistencia que se otorgaba a los jóvenes a partir del PEC, legitimada en términos de su protección, tanto en términos de reparar su vulnerabilidad social, como de paliar su vulnerabilidad penal: alejarlos de los riesgos para sí que implican las prácticas delictivas y ofrecerles otros proyectos de vida alternativos (Ayos, 2013), en los cuales puede vislumbrarse el ideal social de la autovalía, es decir, la organización de las condiciones de vida, la satisfacción de las necesidades en torno al propio trabajo.

Sin embargo, estas intervenciones han mostrado en su desarrollo algunas aristas que corroen dicha forma de justificar la dependencia de los jóvenes en conflicto con la ley penal. Si se observan los "mecanismos preventivos" (Ayos, 2010 y 2013) que estos programas pusieron en marcha, es decir, cuáles eran los encadenamientos causales que las intervenciones suponían, y por tanto en qué sentido estas entendían que eran preventivas del delito, se pueden ver algunas dimensiones que contrastan con aquella "dependencia legítima" que se describió anteriormente. Se hace referencia a dos ejes: la exterioridad e instrumentalidad de la política social, y la noción de transformación subjetiva.

En relación al primero, referido "al lugar" de la política social, surge su tratamiento en términos de su exterioridad e instrumentalidad, en el sentido de no entenderla como parte de la intervención preventiva del delito, sino ubicarla como una herramienta para el ingreso de los jóvenes destinatarios en los dispositivos propuestos en el PCV.

Se hace referencia a la dinámica siguiente: si bien el PCV y el PEC se articulaban y recortaban su población objetivo de manera mutua, la transferencia de ingresos que otorgaba el PEC (la única otorgada a los destinatarios de PCV) era entendida en el desarrollo del PCV como algo exterior a su intervención y tratada sólo en términos instrumentales. El 
PEC es entendido sólo como una herramienta que atrae y hace ingresar a los jóvenes en las actividades que prescribía el PCV, descentrando su valor en relación a su capacidad de transformar las condiciones de vida de dicho grupo.

En el programa nunca fue importante. Siempre ponemos en primera instancia el espacio que brindamos, antes que el PEC [...]. Porque es así, el PEC es una cosa que viene del ministerio, y nuestro programa es otra cosa. Entonces siempre tratamos de separar eso (entrevista personal, 2008a).

Entonces, para nosotros siempre lo que tuvimos en claro es que era una herramienta [...]. La idea es que sirva como una herramienta para acceder a otros aspectos de la vida de ese joven que nos preocupa y creemos que tenemos que trabajar más en ese punto [...] (entrevista personal, 2008b).

A ver, a nosotros nos sirvió como la manzana, para atraerlos, como la zanahoria, digamos. Como decía una compañera mía, es la zanahoria para atraerlos y poder trabajar (entrevista personal, 2008c).

Por ello el lugar de la política social y la capacidad de transformación de las condiciones de vida quedan desdibujadas como "mecanismo preventivo". Y así queda también trastocada aquella orientación que entendía el mejoramiento en las condiciones de vida como una de las justificaciones para el espacio de "dependencia" que elaboraba el programa. Este hincapié en las actividades que organizaba el PCV, en contraposición del beneficio que otorgaba el PEC, se enmarcó en una verdadera crítica a la asistencia, mirando negativamente la "mera" transferencia de ingresos.

Por otro lado, el segundo eje de estos mecanismos preventivos aparece como contracara de esta exterioridad e instrumentalidad de la política social: la idea de una 
transformación subjetiva en términos individuales de los jóvenes destinatarios.

Entonces, por más que venga por el PEC vos lo tenías tendido ahí para laburarlo [sic] y vos tenías que aprovechar esa oportunidad para laburarlo [sic], era lo que vos tenías para agarrarlo y para que venga una vez por semana, dos veces por semana, para moldearlo, para tratar de laburar [sic] con el pibe (entrevista personal, 2008d).

Esta forma de construir los mecanismos preventivos de la intervención presenta dos dimensiones distinguibles aunque encadenadas: por un lado, las operaciones de transformación subjetiva orientadas hacia la producción de límites, en el marcaje de los modos de comportamiento de los jóvenes, centradas en la interpelación a partir de su compromiso con las actividades de los programas.

Por lo general, por mi experiencia acá en este programa, ellos son jóvenes que nunca han tenido límites, de familia, de escuela [...], porque los límites que les han impuesto como, por ejemplo, las normas del colegio, los han abandonado. Yo creo que ya desde marcarle un encuadre desde el programa, de tener que respetar un horario de entrevista, o de grupo, de tener que quedarse ahí sentado una hora en el grupo sin tener que irse, es como que van marcando de a poco unas ciertas normas que por ahí él no vivió en su casa (entrevista personal, 2008a).

Por otro lado, las vinculadas a la constitución de capacidades, como dotación de herramientas subjetivas destinadas a los jóvenes como forma de posibilitar cursos de acción diferentes a los del delito y la violencia.

Desarrollar la capacidad de reconocer puntos de vista diferentes [...], la capacidad de analizar los sucesos en una lógica de causa-efecto concatenados con otros, fomentando así el desarrollo del "locus de control interno"[...]. [Desarrollar la capacidad para] [...] la reconstrucción de 
historias individuales, con miras a elaborar la ubicación temporal-espacial y ubicar diversos factores actuantes en el desarrollo y actualidad de la vida de una persona, así como en la construcción de condiciones y posibilidades de proyección a futuro (PCV, 2005).

En este sentido, se puede observar cómo el lugar subsidiario que es otorgado a la política social en términos de mera instrumentalidad para conformar la población objetivo de los programas preventivos se complementa con la centralidad de la idea de transformación subjetiva que muestra el desarrollo de los mismos. En este punto, puede observarse la tensión entre esta construcción y aquella orientación "social" que pretendía la reparación de las condiciones de vulnerabilidad como forma de protección de jóvenes destinatarios. Aquel espacio de dependencia era justificado en la protección de los derechos de los jóvenes; este se legitima en su capacidad para transformar su subjetividad bajo el supuesto de su dificultad para reconocer los límites y la necesidad para trabajar sobre sus (in)capacidades.

Esta forma de construir la mirada sobre los destinatarios de las estrategias preventivas muestra rasgos de época, subsidiarios con las formas en que son problematizados los territorios y las poblaciones bajo la lógica de los programas asistenciales laborales marcadamente neoliberales. La preocupación por la reactivación de las capacidades, muy fuertemente vinculadas al trabajo y a la cuestión de la posibilidad de estructurar un proyecto de vida racionalmente formulado, atraviesa las modalidades del workfare y de las transferencias monetarias condicionadas que se presentaron en el apartado anterior.

\section{Conclusiones}

Este trabajo se ha propuesto analizar las formas en que los programas de prevención social del delito participan 
de la producción social sobre las figuras de la autovalía o la dependencia legítima con respecto a los jóvenes que son destinatarios de sus intervenciones. Y, en este sentido, la propuesta solapaba, por un lado, la preocupación por las transformaciones en torno al trabajo, las condiciones de vida y sus condiciones socioinstitucionales, y, por el otro, los debates que atraviesan a la sociedad argentina sobre la cuestión de la (in)seguridad y el delito, especialmente en referencia a los jóvenes de sectores populares como figuras de la peligrosidad.

Los programas de prevención social del delito constituyeron un espacio de articulación y tensión de estas dos líneas de fuerza, expresando a su vez las reorientaciones de sus respectivos campos de intervención: la política social y la política criminal. En este sentido, se ha mostrado cómo los programas de prevención social pueden observase como el encuentro entre la asistencialización del campo de la política social, enfatizando la preocupación por las capacidades individuales sobre la problematización de lo social en el marco de un proceso de individualización, y como una orientación "progresista" dentro del campo del control del delito, que entendió que la propuesta por una "política democrática de seguridad" debía materializarse en un estrategia preventiva que incidiera en las supuestas "causas sociales" del delito.

El declive de esta articulación estratégica se produjo en el contexto de la reconfiguración entre los sectores de la asistencia y la seguridad social que marcara la emergencia de la AUHPS, produciendo un fuerte debilitamiento de la corriente de los programas tipo workfare, con "contraprestación" laboral, y, a la vez, como parte de una transformación en la noción de seguridad democrática, que a partir de la creación del Ministerio de Seguridad se entendió fundamentalmente desde la cuestión del control político de las fuerzas de seguridad, descentrando el eje de la prevención "social" del delito. 
En este desarrollo, las políticas de prevención social tensionaron figuras diferentes de la autovalía y la dependencia con respecto a los jóvenes destinatarios. Por un lado, reconstruyeron un espacio de dependencia legítima al entender a estos programas como ámbito de protección de los derechos de los jóvenes que se veían amenazados tanto por sus condiciones de vida como por su vulnerabilidad frente a las agencias penales. Sin embargo, en línea con las transformaciones analizadas en el campo de las políticas sociales, el descentramiento de la idea de transformación de las condiciones de vida y la fuerte preocupación por las capacidades subjetivas de estos jóvenes marcan una orientación distinta en la problematización sobre las responsabilidades sociales. Aquel espacio de dependencia legítima en referencia a las nociones de derechos y protección se desdibuja en formas de responsabilización individual centrada en la problemática de las capacidades subjetivas.

Se entiende que esta caracterización histórica del devenir de los programas de prevención del delito y de las formas de problematización y tratamiento de los jóvenes de sectores populares en Argentina constituye un necesario punto de partida para interrogar sobre el contexto actual, caracterizado en principio por el declive de este tipo de intervención preventiva sobre jóvenes. Se considera que las claves que marcan este presente se tensionan entre el avance de la lógica de la seguridad social en el campo de las intervenciones sociales, incluso sobre la población juvenil, y la persistente presencia de un fuerte punitivismo en las discusiones sociales sobre la cuestión de la (in)seguridad en el que dificultosamente navegan los proyectos por una seguridad democrática. 
Bibliografía

Álvarez Leguizamón, S. (2006). “La invención del desarrollo social en la Argentina: historia de 'opciones preferenciales por los pobres"', en L. Andrenacci (comp.), Problemas de política social en la Argentina contemporánea (pp. 8I-124). Buenos Aires: Prometeo-UNGS.

Ayos, E. (2010). Delito y pobreza: espacios de intersección entre la política criminal y la política social argentina en la primera década del nuevo siglo. Sao Paulo: IBCCRIM.

(20I3). "La prevención como contraprestación: la instrumentalidad de la asistencia en la intersección de la política social y la política criminal". Revista Trabajo y Sociedad, (2I), 225-246.

(20I4). “¿Una política democrática de seguridad? Prevención del delito, políticas sociales y disputas en el campo conformado en torno a la inseguridad en la Argentina de la última década". Revista del CLAD Reforma y Democracia, (58), 169-200.

(07 de mayo de 2008a). Entrevista personal realizada a Silvia, operadora Programa Comunidades Vulnerables. Buenos Aires, Argentina.

(20 de mayo de 2008b). Entrevista personal a Beatriz, operadora Programa Comunidades Vulnerables. Buenos Aires, Argentina.

(03 de junio de 2008c). Entrevista personal a Lorena, operadora Programa Comunidades Vulnerables. Buenos Aires, Argentina.

(24 de septiembre de 2008d) Entrevista personal realizada a Claudia, Autoridad Local Programa Comunidades Vulnerables. Buenos Aires, Argentina.

Baratta, A. (1986). Criminología crítica y crítica del derecho penal. Buenos Aires: Siglo XXI.

(1997). "Política criminal: entre la política de seguridad y la política social”, en E. Carranza (coord.), Delito y seguridad de los habitantes (PP. 80-96). México: Siglo XXI. 
Bauman, Z. (1999). La globalización. Consecuencias humanas. Buenos Aires: Fondo de Cultura Económica.

Beck, U. (1998). La sociedad del riesgo. Hacia una nueva modernidad. Barcelona: Paidós.

Boutellier, H. (200I). "The convergence of social policy and criminal justice". European Journal on Criminal Policy and research, 9, 36I-380.

Calzado, M. (2004). “Discursos sociales y prácticas punitivas. Las construcciones significantes de la opinión pública en las asambleas del Plan de Prevención del Delito". Revista Delito y Sociedad, (20), I37-I57.

— y Van Den Dooren, S. (2009). "¿Leyes Blumberg? Reclamos sociales de seguridad y reformas penales". Revista Delito y Sociedad, (27), 97-II 3.

Castel, R. (1997). Las metamorfosis de la cuestión social. Una crónica del salariado. Buenos Aires: Paidós.

- (2004). La inseguridad social. ¿Qué es estar protegido? Buenos Aires: Manantial.

Ciafardini, M. (2006). Delito urbano en la Argentina. Las verdaderas causas y las acciones posibles. Buenos Aires: Ariel.

Cohen, S. (1985). Visiones del control social. Barcelona: Ediciones PPU.

Cortés, R., y Marshall, A. (1999). "Estrategia económica, instituciones y negociación política en la reforma social de los '90". Revista Desarrollo Económico, (I54), 195-2I4.

Crawford, A. (1998). Crime Prevention and Community Safety. Politics, Policies and Practices. Harlow: Longman. (2009). "Situating crime prevention policies in comparative perspective: policy travels, transfer and translation", en A. Crawford (ed.), Crime Prevention Policies in Comparative Perspective (pp. I-37). Devon: Willan Publishing.

Danani, C. (2004). "El alfiler en la silla: sentidos, proyectos y alternativas en el debate de las políticas sociales y de la economía social. Introducción”, en C. Danani (comp.), 
Política social y economía del trabajo (pp. 9-38). Buenos Aires: UNGS/OSDE/Altamira.

(2005a). "Las políticas sociales de los '90: los resultados de la combinación de individualización y comunitarización de la protección”, en, Coloquio internacional: trabajo, conflictos sociales e integración monetaria: América Latina en una perspectiva comparada (pp. 14-26). Buenos Aires: Instituto de Ciencias (UNGS)/Institut de Reserche por le Développement/ANPCYT-FONCYT.

(20I0). "El espacio público en su laberinto: las políticas sociales y las confrontaciones del universalismo", en N. Gluz, y J. Arzate Salgado (comps.), Particularismo y universalismo en las políticas sociales: el caso de la educación (pp. 65-86). Buenos Aires: UNGs-Universidad Autónoma del Estado de México.

- y Grassi, E. (2008). "Ni error ni omisión. El papel de la política de Estado en la producción de las condiciones de vida y de trabajo. El caso del sistema previsional, Argentina 1993-2008", en J. Lindenboim (comp.), Trabajo, ingresos y políticas en Argentina. Contribuciones para pensar el siglo XXI (pp. 259-298). Buenos Aires: Editorial Eudeba.

- y Hintze, S. (20I I). "Reformas y contra-reformas de la protección social: la seguridad social en la Argentina en la primera década del siglo”. Revista Reflexión Política, 12, 18-29.

Daroqui, A. (2003). "Las seguridades perdidas”. Argumentos, Revista Electrónica del Instituto de Investigaciones Gino Germani, (2), s/p. Recuperado de: http://publicaciones. sociales.uba.ar/index.php/argumentos/article/view/8I 5 (2009). Muertes silenciadas: la eliminación de los "delincuentes". Una mirada sobre las prácticas y los discursos de los medios de comunicación, la policía y la justicia. Buenos Aires: Ediciones del CCC. 
y Guemureman, S. (200I). La niñez ajusticiada. Buenos Aires: Editores del Puerto.

Bibliografía

y Guemureman, S. (2004). "Políticas penales de seguridad dirigidas hacia adolescentes y jóvenes en la década del '90 en la Argentina", en I. Muñagorri, y J. Pegoraro (comps.), La relación seguridad-inseguridad en centros urbanos de Europa y América Latina (pp. 123- I40). Madrid: Dykinson.

De Marinis, P. (1998). "La espacialidad del ojo miope (del poder). (Dos ejercicios de cartografía postsocial)". Archipiélago, cuadernos de crítica de la cultura, (34-35), 32-39.

- (2002). "Ciudad, 'cuestión criminal' y gobierno de poblaciones”. Revista Política y Sociedad, 39(2), 319-338.

Font, E. (1999). "Transformaciones en el gobierno de la seguridad: análisis exploratorio de conceptos y tendencias. Su relevancia en Argentina”, en M. Sozzo (comp.), Seguridad urbana: nuevos problemas, nuevas perspectivas. Pensar alternativas teóricas y políticas sobre la cuestión criminal (pp. 89-II8). Santa Fe: UNL.

Foucault, M. (1999). Vigilar y castigar. México: Siglo XXI.

Galvani, M., et al. (20I0). A la inseguridad la hacemos entre todos. Prácticas académicas, mediáticas y policiales. Buenos Aires: Hekht Editores.

Garland, D. (1985). Punishment and welfare: a history of penal strategies. Aldershot: Grower.

- (2005). La cultura del control. Crimen y orden social en la sociedad contemporánea. Barcelona: Gedisa.

Glaser, B. G., y Strauss, A. L. (1967). The discovery of grounded theory. Nueva York: Aldine.

Grassi, E. (2003). Política y problemas sociales en la sociedad neoliberal. La otra década infame. Buenos Aires: Espacio Editorial.

Hintze, S., Neufeld, M., et al. (1994). Políticas sociales, crisis y ajuste estructural. Buenos Aires: Espacio Editorial. 
Bibliografía

Grondona, A. (2012). "Tradición" y "traducción": un estudio de las formas contemporáneas del gobierno de las poblaciones desempleadas en la Argentina. (tesis de doctorado inédita). Universidad de Buenos Aires: Buenos Aires. Recuperado de: http://www.centrocultural.coop/ descargas/tesis/tradicion-y-traduccion-un-estudiode-las-formas-contemporaneas-del-gobierno-de-laspoblaciones.html

Guemureman, S. (2002). "La contracara de la violencia adolescente-juvenil: la violencia pública institucional de la agencia de control social judicial", en S. Gayol, y G. Kessler (comps.), Violencias, justicias y delitos en la Argentina (pp. 169-189). Buenos Aires: Ediciones Manantial y Universidad Nacional de General Sarmiento.

Hintze, S., y Costa, M. (20I I). "La reforma de las asignaciones familiares 2009: aproximación al proceso político de la transformación de la protección", en C. Danani, y S. Hintze (coords.), Protecciones y desprotecciones (pp. 153-183). Los Polvorines: UNGS.

(20I4). "Capacidad protectoria de la Asignación Universal por Hijo para Protección Social: problemas y debates a cuatro años de implementación", en C. Danani, y S. Hintze (coords.), Protecciones y desprotecciones (II): problemas y debates de la seguridad social en Argentina (Pp. 243-280). Los Polvorines: UNGS.

Isla, A. (2002). "Los malvivientes", en S. Gayol, y G. Kessler (eds.), Violencias, delitos y justicias en la Argentina (pp. 295-3 I3). Buenos Aires: UNGS/Manantial.

- (2007). En los márgenes de la ley: inseguridad y violencia en el cono sur. Buenos Aires: Paidós.

Kaminsky, G. (2005). "Territorios inseguros, estigmas ciudadanos”, en G. Kaminsky (aut.), Tiempos inclementes. Culturas policiales y seguridad ciudadana (pP. 16-64). Buenos Aires: UNLA. 
Kessler, G. (2002). "Entre fronteras desvanecidas. Lógicas Bibliografía de articulación de actividades legales e ilegales en los jóvenes", en S. Gayol, y G. Kessler (eds.), Violencias, justicias y delitos en la Argentina (pp. 339-355). Buenos Aires: Ediciones Manantial y Universidad Nacional de General Sarmiento.

(2006). Sociología del delito amateur. Buenos Aires: Paidós. (2008). "Delito, trabajo y ley”. Revista Puentes, (23), 83-95.

Lea, J., y Young, J. (2008). ¿Qué hacer con la ley y el orden? Buenos Aires: Editores del Puerto.

López, A., et al. (20I I). "Estrategias de gobierno del territorio urbano: hostigamiento y brutalidad policial sobre los jóvenes en la provincia de Buenos Aires" (trabajo presentado en la mesa de trabajo sobre "Sistema Penal y DDHH" de las IX Jornadas de Sociología). Buenos Aires: Universidad de Buenos Aires.

Lo Vuolo, R. (2009). Asignación por hijo. Buenos Aires: CIEPP. Melossi, D., y Pavarini, M. ( 1987). Cárcel y fábrica: los orígenes del sistema penitenciario. México: Siglo XXI.

— y Selmini, R. (2009). “'Modernisation' of institutions of social and penal control in Italy/Europe: the 'new' crime prevention”, en A. Crawford (ed.), Crime Prevention Policies in Comparative Perspective (PP. 153- I76). Devon: Willan Publishing.

Míguez, D. (2002). "Los rostros del desorden. Fragmentación social y la nueva cultura delictiva en sectores juveniles”, en S. Gayol, y G. Kessler (eds.), Violencias, delitos y justicias en la Argentina (pp. 314-338). Buenos Aires: UNGS/Manantial.

MTEYSS (2003). Creación del Programa de Empleo Comunitario. Resolución Nacional 7/2003. Buenos Aires: Ministerio de Trabajo y Seguridad Social de la Nación. 
Bibliografía
Murillo, S. (2008). Colonizar el dolor. La interpelación ideológica del Banco Mundial en América Latina. El caso argentino desde Blumberg a Cromañón. Buenos Aires: CLACSO.

O'Malley, P. (2004). "Riesgo, poder y prevención del delito". Revista Delito y Sociedad, (20), 79- 102.

- y Hutchinson, S. (2007). "Reinventing Prevention: Why Did 'Crime Prevention' Develop So Late?’. British Journal of Criminology, 47(3), 437-454.

Offe, C. (1990). "La política social y la teoría del Estado”. En C. Offe (aut.), Contradicciones en el Estado de bienestar (pp. 72-104). Madrid: Alianza.

Pavarini, M. (1983). Control y dominación. México: Siglo XXI. Pegoraro, J. (200I). "Inseguridad y violencia en el marco del control social”. Espacio Abierto, Cuaderno Venezolano de Sociología, I0(3), 349-372.

- (2002). "Teoría sociológica y delito organizado: el eslabón perdido”. Encrucijadas UBA. Revista de la Universidad de Buenos Aires, (19), 38-55.

PCV (2005). Sistematización Taller de proyecto de vida e identidad. Documento $N^{\circ} 7$. Argentina: Programa Comunidades Vulnerables, Dirección Nacional de Política Criminal, Ministerio de Justicia y Derechos Humanos. PIM (2008) Programa Intervención multiagencial para el abordaje del delito en el ámbito local. Argentina: Secretaría de Seguridad Interior, Ministerio de Justicia y Derechos Humanos.

Pitch, T. (2009). La sociedad de la prevención. Buenos Aires: Ad Hoc.

Rangugni, V. (2009). "Emergencias, modos de problematización y gobierno de la in/seguridad en la argentina neoliberal". Delito y Sociedad. Revista de Ciencias Sociales, (27), 23-43.

Rose, N. (1996). "The death of the social? Re-figuring the territory of government". Economy and Society, (25), 327-356. 
Rossini, G. (2003). "Vagos, pibes chorros y transformaciones de la sociabilidad en tres barrios periféricos de una ciudad entrerriana”, en A. Isla, y D. Mígues (coords), Heridas urbanas. Violencia delictiva y transformaciones sociales en los noventa (Pp. 7I-II5). Buenos Aires: Editorial de las Ciencias-FLACSO Argentina.

Saín, M. (2002). Seguridad, democracia y reforma del sistema policial en la Argentina. Buenos Aires: Fondo de Cultura Económica.

(2008). El Leviatán Azul. Buenos Aires: Siglo XXI Editores.

(20I0). "Progresismo ficcional. La política de seguridad pública durante la gestión presidencial de Néstor Kirchner (2003-2007)" (ponencia presentada en las VI Jornadas de Sociología). Buenos Aires: Universidad Nacional de La Plata.

Selmini, R. (2009). "La prevención: estrategias, modelos y definiciones en el contexto europeo". Urvio. Revista Latinoamericana de Seguridad Ciudadana, (6), 4I-57.

Soldano, D., y Andrenacci, L. (2006). "Aproximación a las teorías de la política social a partir del caso argentino", en L. Andrenacci (comp.), Problemas de política social en la Argentina contemporánea (pp. 17-80). Buenos Aires: Prometeo/UNGS.

Soneira, A. J. (2004). "La teoría fundada en los datos. Grounded Theory de Glaser y Strauss", en AA. VV., IV Jornadas de etnografia y métodos cualitativos (Pp. 83-97). Buenos Aires: IDES.

Sozzo, M. (1999). Seguridad urbana: nuevos problemas, nuevos enfoques. Santa Fe: UNL.

(2000). "Seguridad urbana y técnicas de prevención del delito". Cuadernos de Jurisprudencia y Doctrina Penal, (10), 17-82.

(2008). Inseguridad, prevención, policía. Quito: FLACSO Ecuador. 
Bibliografía

Tonkonoff, S. (2003). “Microdelitos, juventudes y violencias: la balada de los pibes chorros". Revista Delito y Sociedad, (18-19), 109-1 23.

(2007a). "Tres movimientos para explicar por qué los pibes chorros visten ropas deportivas", en AA. VV., La sociología ahora (pp. 5-I5). Buenos Aires: Siglo XXI Editores.

(2007b). Juventud, exclusión y delito. Notas para la reconstrucción de un problema. Recuperado de: http:// www.pensamientopenal.com.ar/0 I0320 I0/criminologia0l.pdf

Vilker, S. (20II). "No hay solución. Un estudio de recepción de noticias sobre juventud y delito del repertorio cultural de la víctima al nihilismo propositivo", en M. Gutiérrez (comp.), Populismo punitivo y justicia expresiva (pp. I2 I- I43). Buenos Aires: Fabián J. Di Plácido Editor. Wacquant, L. (2000). Las cárceles de la miseria. Buenos Aires: Manantial. 